\title{
L'expression théâtrale en FLE : en route vers une cocréativité !
}

\section{Marie-Noëlle COCTON}

CIDEF, Université Catholique de l'Ouest, Angers ; Licia - CoDiRe, Université de Nantes, France

\section{Introduction}

Susciter l'émergence du désir de parole du sujet par une relation subjectivante et interculturelle novatrice entre parole, corps et culture(s) est un objectif pédagogique et une quête didactique inhérents au travail d'enseignement d'une langue qui tient compte de la pluralité des contextes et de la singularité de chacun. C'est ce vers quoi tend la pratique théâtrale destinée aux apprenants de français langue étrangère. Sa mise en œuvre - qui a accompagné la fondation des filières de FLE dans certaines universités françaises au début des années quatre-vingts - a entrainé, ces dernières années, de nombreuses réflexions ${ }^{1}$ sur la place du théâtre dans l'enseignement d'une langue étrangère et ce, grâce au développement des approches communicatives et actionnelles dans la didactique des langues. Quels rôles joue le théâtre en didactique des langues ? Quels en sont les bienfaits pour l'apprenant? N'est-ce pas dévaloriser le théâtre que de le réduire à un simple outil pédagogique ?

L'objectif de cet article n'est pas de répondre à ces questions mais de partager une expérience pédagogique menée en classe de FLE auprès d'un public d'apprenants de niveau de langue B1. Avant de donner quelques précisions sur la méthodologie adoptée, j'expliquerai, dans une première partie, ce qui est entendu par «expression théâtrale » et les raisons qui ont conduit à recourir à cette pratique. Enfin, je donnerai les bienfaits tant au niveau pédagogique que personnel de l'action théâtrale afin de mieux apprécier les notions de « cocréativité » et «co-cultures ».

\section{1- Le projet pédagogique}

\section{1- Constats}

Dans la perspective actionnelle esquissée par le Cadre européen commun de référence pour les langues ${ }^{2}$, on se propose de former un «acteur social», ce qui devrait impliquer, selon Christian Puren, « de le faire agir avec les autres pendant le temps de son apprentissage en lui proposant des occasions de "coactions" dans le sens d'actions communes à finalité collective » (65). Puisqu'elle ne fait plus appel au «parler sur des documents » comme dans 
la méthodologie directe ou au "parler avec des interlocuteurs » comme dans l'approche communicative, cette démarche invite à la réflexion didactique : comment proposer des occasions d'enjeu social authentique en classe ? Quelles activités permettraient de dépasser la reproduction de la réalité - autrement dit, la simulation des années 1970 - pour créer non plus des situations de simple interaction langagière mais des possibilités de coactions langagières ?

Pour répondre à ces questions, il convient de s'interroger sur les activités proposées dans les manuels de FLE ou dans les examens de type DELF-DALF afin de mieux comprendre l'enseignement-apprentissage de la «production orale » depuis la mise en place du CECR.

Quelques constats :

- Il n'existe pas toujours dans les manuels de langue d'activités orales autres que des interactions par paires, des questions fermées et des échanges en classe.

- Les épreuves de production orale fournies dans le cadre d'une préparation au DELF se construisent autour d'un entretien dirigé, d'un exercice en interaction et de la présentation d'un point de vue. Ces activités de face à face ne reflètent pas la réalité des situations de communication langagière où la construction communicative s'effectue à l'aide des apports de multiples interactants et où les interruptions sont nombreuses.

- L'évaluation des productions orales est, encore aujourd'hui, problématique. Les grilles proposées dans les guides pédagogiques sont, souvent, assez éloignées de la réalité de l'interaction et nécessiteraient un matériel d'enregistrement permettant de capturer l'image et le son afin d'évaluer l'ensemble des compétences mobilisées qui assurent à la performance sa qualité.

- Les étudiants apprennent une langue décorporalisée (Caré 48). Quand on demande à un enseignant quelle position il adopte quand il enseigne, il répond généralement « debout». Ce choix n'est probablement pas fortuit. Mais demande-t-on à un apprenant dans quelle position il apprend le mieux ?

- Les apprenants ont rarement la possibilité de communiquer avec leur corps. Pourtant, il suffit d'observer une interaction entre apprenants d'origines ethniques différentes pour se rendre compte que chacun a son propre langage du corps. Un asiatique sera probablement plus économe de ses gestes qu'un occidental ou qu'un latino-américain, mais non moins signifiant dans son syntagme corporel. 
- Le cours de langue n'est pas toujours un lieu où la communication orale prend place aisément : les apprenants sont parfois trop nombreux pour permettre un temps de parole régulier et la relation didactique enseignant-apprenant est asymétrique. Cette asymétrie peut entrainer quelques blocages ou difficultés à la prise de parole.

\section{2- Le cours d'expression théâtrale}

C'est le désir de mettre l'apprenant en action en lui faisant prendre conscience de ses ressources langagières en jeu qui a contribué à nous orienter vers la mise en place d'un enseignement de la compétence orale en choisissant une didactique du FLE alternative. Cette approche s'appuie sur des techniques largement utilisées dans d'autres domaines, ici principalement le théâtre, sans pour autant leur accorder un rôle exclusif ou prétendre nécessairement à des représentations sur scène. Dans un souci de communication «multicanale », nous avons fixé des objectifs tels que découvrir ou redécouvrir sa voix en langue étrangère, apprendre à utiliser les rythmes et intonations adaptés à chaque situation à travers des exercices de respiration, de sonorité, de lecture expressive, un travail sur le silence et les pauses. Découvrir son corps ou le redécouvrir à travers des déplacements dans l'espace, des précisions dans les gestes, des émotions sur le visage. Délaisser la langue pour faire place au non verbal, au corps poétique. Apprendre ou réapprendre à écouter l'autre, avec ses oreilles et aussi ses yeux, lui répondre de façon immédiate, spontanée et engagée.

Pour mener à bien ce projet, il semblait nécessaire que ce cours :

- n'accueille pas plus de douze étudiants pour permettre un véritable échange, une individualisation et une correction langagière ${ }^{3}$;

- reste une option que les apprenants sont libres de choisir ;

- fonctionne par niveau : les étudiants sont de niveau $\mathrm{A} 2$ à $\mathrm{C} 2$, avec un programme adapté à chaque niveau ;

- soit, presque exclusivement, un cours «debout». Les cours se passent dans une salle de classe mais les chaises et les tables sont mises de côté. La communication invite à jouer avec l'espace, le corps et les mouvements.

\section{2- Méthodologie}

\section{1- Les objectifs du cours}

L'objectif principal est de mener l'apprenant à une expression spontanée et créative en construisant avec l'autre de façon imprévisible. Cette démarche suppose certains choix pédagogiques et méthodologiques. Pour ce faire, avec une collègue, nous nous appuyons sur : 
- notre propre formation théâtrale, en tant qu'artistes amateurs,

- des activités proposées par des professionnels du théâtre comme Jacques Lecoq, Augusto Boal, Constantin Stanislavski,

- les travaux réalisés par le Programme Socrates notamment, le cahier d'exercices, Le français par les techniques théâtrales,

- des manuels divers comme celui d'Adrien Payet, de Christophe Tournier, de Michèle Vielmas et bien d'autres.

Le cours, d'une durée d'environ trente-six heures par semestre, est divisé en trois parties : un travail sur le «moi-même », «l'autre » et le « jouer à être un autre».

La première étape est essentielle: elle part du principe que lorsque nous communiquons, nous ne sommes que peu conscients de ce qui constitue notre «parler». Les recherches qui existent depuis plus de cinquante ans sur la communication ont et ce, grâce à l'école Palo Alto, affirmé que «la communication est un processus social permanent intégrant de multiples codes de comportement: la parole, le geste, le regard, la mimique, l'espace interindividuel, etc. » (Winkin 24). Dans son apprentissage de la langue étrangère, l'apprenant fait généralement attention à la manière dont il s'exprime verbalement mais ne cherche pas à observer ce que son corps, sa voix et ses gestes véhiculent. Un des objectifs est donc de lui faire apprécier sa voix en langue étrangère, en lui apprenant à utiliser les rythmes et intonations adaptés à chaque situation à travers des exercices de respiration, de sonorité, un travail sur le silence, les pauses et les effets de rythme. Un travail d'exploitation du corps et des émotions est également proposé et ce, afin de conduire l'apprenant vers une expression personnelle globale. Ces activités portent sur les mimiques (ex : le jeu du miroir), le regard (ex : regarder son auditoire en entrant sur scène), les émotions (ex : le masque), les gestes (ex : raconter une histoire sans parole), l'espace (ex : marcher dans une forêt, dans la mer, dans la boue, sur la neige...), la voix (ex : mettre en voix un texte écrit par l'apprenant à partir d'un poème), etc.

Une fois que les apprenants ont porté un premier regard sur leurs propres capacités ou difficultés (ex : difficulté pour un apprenant japonais d'imaginer, pour un apprenant canadien de parler lentement, pour un étudiant coréen de parler fort, etc.), chacun sera prêt à travailler avec «l'autre». Des activités en binômes tels que les mimes, les disputes, les répliques immédiates (ex : «le jeu du goaler»), les jeux de persuasion, invitent chacun à prendre connaissance du geste, de l'imaginaire, des expressions, des mimiques, des idées, des 
arguments de l'autre auxquels il n'aurait pas pensé. C'est un véritable partage de compétences qui s'opère alors, chacun s'enrichissant par le biais de son partenaire.

Vient enfin le changement personnel de statut. «Jouer à être un autre » s'accomplit à travers des activités de dynamisation (ex : les démarches), de construction (ex : le scénario à plusieurs), d'improvisations libres ou contextualisées. L'apprenant est invité à jouer une multitude d'identités, de sorte qu'il rentre dans l'imaginaire d'un autre sans pour autant le stéréotyper. Pour cela, on le fait travailler autour de ce qu'il vit en France, en lui demandant de prendre le rôle d'une personne qu'il a rencontrée (ex: dans sa famille d'accueil) en s'attachant à être précis, clair et harmonieux de façon visuelle et verbale. L'objectif est de vérifier la cohérence des énoncés avec le statut du personnage. En multipliant les contextes d'action (ex : à la gare, à la piscine, au musée), on développe un choix d'énoncés possibles. On peut envisager le même type de travail avec des personnages éloignés de la réalité quotidienne des étudiants (ex : cowboy, princesse, pirate, etc.). «Jouer à être un Français » permet de répondre aux questions culturelles ${ }^{4}$ que l'apprenant se pose.

\subsection{Le déroulement d'une séance}

Généralement, une séance dure cinquante-cinq minutes. Le cours s'organise de la manière suivante :

- brise-glace (5 minutes)

- échauffement (15 minutes)

- jeu théâtral (25 minutes)

- échange (10 minutes)

Le brise-glace a pour fonction de dynamiser la classe en créant une cohésion de groupe où chacun trouvera un certain plaisir à jouer et à s'exprimer en langue étrangère. «Les jeux de dynamisation permettent de stimuler la prise de parole en français tout en créant une atmosphère ludique » (Payet 25). Ces jeux tels que «le passage d'énergie » ou «la machine infernale » permettent à chacun de prendre le temps de se resituer dans le cours, d'être en confiance avec le groupe et de rassembler et focaliser son énergie en vue d'une concentration ultérieure.

Suivent les activités d'échauffement dont le but est de rendre l'apprenant le plus ouvert et le plus disponible aux activités suivantes. Issus du théâtre, ces exercices partent du non verbal, continuent avec l'émission vocale et finissent par le verbal. Le répertoire est riche. Peuvent être évoqués : le travail de respiration qui chauffe les muscles, celui sur 
l'articulation qui mobilise les lèvres et la mâchoire inférieure, celui sur la pose de la voix et l'intensité vocale, celui sur le silence, animé par la concentration et la force du regard.

Les apprenants sont ensuite invités à constituer de petits groupes (3 à 4 personnes) afin de préparer un jeu dramatique. Une consigne leur est donnée oralement. Exemple : «Vous êtes assis sur un banc dans un parc. Vous jouez un personnage imaginaire. Un évènement inattendu va se produire. Organisez votre improvisation en commençant par penser à la chute. Vous avez deux minutes de préparation ». Cette activité est faite des compétences de chaque apprenant qui, individuellement, va contribuer à la coconstruction d'une mini improvisation en apportant de son savoir, de son savoir-faire et de son savoir-être. L'un aura peut-être plus d'imagination, l'autre plus de dynamisme, l'un plus de spontanéité, l'autre plus de répartie. Les apprenants peuvent avoir des idées d'action différentes ou des moyens d'actions différents. La coaction met les compétences de chacun en commun pour aboutir à un résultat.

Les activités peuvent être variées : jeu d'expression mimique et gestuelle, avec ou sans paroles, à partir d'une émotion ou d'un sentiment, jeu de mimes à répétitions, improvisation à partir d'un document déclencheur, dramatisation à partir d'un texte, de faits divers ou d'articles de presse, travail de lecture expressive. Pendant le jeu, les apprenants sont sollicités pour construire un personnage, lui donner un corps, le faire vivre dans ses relations avec les autres, lui faire ressentir des émotions toutes particulières. Ce travail, qui peut se faire avec ou sans masques, renvoie au modèle historique de la Commedia Dell'Arte. Au départ, les gestes et les paroles étaient inventés en coulisses à partir de canevas. Le spectacle consistait en une rencontre de personnages-types représentant des scènes de la comédie humaine caricaturée. Dans la seconde moitié du seizième siècle, la comédie italienne a confectionné des masques afin de transformer le personnage qui devenait alors un inconnu aux yeux du public. L'apprenant, derrière son masque, devient lui aussi « inconnu » et adopte plus facilement une autre identité.

La dernière étape du cours consiste en un temps d'échange libre ou semi-guidé. Après chaque activité, les apprenants prennent le temps de se poser et de porter un regard extérieur sur ce qu'ils viennent de faire. La réflexion porte autant sur la nature des activités que sur les difficultés rencontrées. Les apprenants échangent autour des intérêts et des objectifs de l'activité, de la réussite ou de l'échec d'un jeu, de la capacité ou non à créer tel ou tel personnage, de l'obstacle de la langue, de l'attitude de tel ou tel apprenant dans son apprentissage et des améliorations à envisager. L'objectif reste la critique constructive : 
positive ou négative, elle doit mener l'apprenant à réfléchir sur sa propre progression et lui apporter conseils et encouragements pour donner qualité à son expression orale, vocale et verbale.

\section{3- Les bienfaits de l'expression théâtrale}

\section{1- Au niveau pédagogique}

Derrière le changement de désignation (de la compétence de communication à la compétence à communiquer langagièrement) se profile un déplacement de paradigme : la notion de compétence renvoie à «la manière dont un individu mobilise de telles capacités (cognitives) et diverses autres ressources cognitives dans des situations complexes, qui exigent l'orchestration de multiples opérations pour résoudre un problème, prendre une décision, conduire une activité, etc. » (Perrenoud). À travers cette définition, on constate que le concept de compétence est lié à celui d'action, de résolution de problèmes, de maitrise de tâches et à la synchronisation de multiples ressources pour réaliser une action finalisée. L'agir serait au cœur de l'apprentissage. À quoi pourrait servir d'apprendre une langue étrangère si ce n'est pour s'exprimer et agir sur autrui ? «Ce n'est pas le système de la langue qui s'apprend mais l'action d'agir sur autrui par le langage en langue étrangère, que celui-ci soit naturel ou esthétique » (Pierra 34). À la différence de Gisèle Pierra, notre travail ne porte par sur l'esthétisme ${ }^{5}$ du texte et de la langue mais sur la « rentabilité communicationnelle », sur la capacité de l'apprenant à communiquer dans l'immédiateté, à réagir au dire d'autrui. Il s'agit, à travers l'utilisation de jeux dramatiques, de développer l'agir-spontané de l'apprenant. Dans le CECR, la compétence pragmatique, composante de la compétence à communiquer langagièrement, traite de la connaissance que l'apprenant a des principes selon lesquels les messages sont utilisés pour la réalisation de fonctions communicatives. Cette même compétence peut-être évaluée de façon qualitative (100) par la capacité de l'apprenant à formuler, poursuivre, résoudre un problème avec aisance et spontanéité. La spontanéité peut être envisagée dans le sens d'aisance à l'oral (compétences énonciatives) et dans le sens d'action volontaire (du latin, sua sponte). L'action volontaire est observable dans le degré d'engagement. L'hypothèse, validée dans un travail de thèse ${ }^{6}$ pour le doctorat, s'appuyant sur les travaux de Tabensky, consiste à considérer l'engagement comme clé dans la construction d'une communication dans la mesure où il participe à l'émergence de la spontanéité (ouvrir ou clore une conversation, tenir ou non un rôle dominant, masse de parole, rythme de l'interaction, interruption volontaire ou non, répliques immédiates, reformulation...). 


\section{2- Au niveau personnel}

Il est intéressant d'observer les raisons qui poussent les apprenants à choisir cette option. Au début du cours, ceux-ci avouent s'y être inscrits parce que «des amis leur ont dit que c'est amusant», par «curiosité », pour «parler autrement», ou parce qu'ils «sont timides ». Puisqu'il s'agit de faire apprendre à communiquer autrement, cela suppose, avant tout, de faciliter la confiance que l'apprenant peut avoir à communiquer. Ce qui ressort de cette expérience est l'investissement personnel des apprenants et le développement d'une certaine confiance. À chaque fin de semestre, les étudiants sont invités à évaluer le cours. Une des questions est : «En général, qu'est-ce que ce cours vous a apporté ?». Sur les dixhuit questionnaires reçus à la fin du semestre de printemps 2011, 15 étudiants répondent « la confiance »: «la confiance dans la langue française, la confiance d'être créatif et de se transformer en une personne qui n'est pas timide devant un public; il m'a apporté un niveau de français à l'oral qui est plus à l'aise, plus spontané ; la confiance et j'ai vraiment bien appris à démontrer mes émotions avec et sans la langue ».

Ce qui ressort des témoignages, est aussi la découverte par l'apprenant de sa propre créativité. Telle qu'elle est définie dans le courant cognitif, celle-ci renvoie à « la capacité à réaliser une production qui soit à la fois nouvelle et adaptée au contexte dans lequel elle se manifeste » (Lubart 10). Dans le champ de l'improvisation, la créativité se manifeste parce que la production est nouvelle - par rapport à ce que le sujet ou d'autres personnes ont déjà réalisé ou qu'elle présente une déviation minime par rapport aux réalisations antérieures - et qu'elle est adaptée, c'est-à-dire qu'elle satisfait différentes contraintes liées aux situations dans lesquelles se trouvent les personnes. Un apprenant ne pourra jamais exactement savoir ce que va faire, dire, penser l'autre au cours d'une action. Il doit l'écouter, s'adapter à lui, être capable d'anticiper ses actes pour créer le scénario en direct. Il ne se contente pas de réagir à l'autre dans un schéma de «stimulus-réponse » : il pro-agit en inventant des solutions au fur et à mesure qu'un scénario se déroule en fonction d'un objectif donné dans un contexte particulier. L’improvisation devient une cocréativité.

\section{3- Au niveau culturel}

Le CECR souligne un des objectifs de l'acquisition d'une compétence interculturelle pour les apprenants: «Dans une approche interculturelle, un objectif essentiel de l'enseignement des langues est de favoriser le développement harmonieux de la personnalité de l'apprenant et de son identité en réponse à l'expérience enrichissante de l'altérité en matière de langue et de culture » $(9)$. 
L'apprenant est invité à observer et repérer le culturel dans la communication ; analyser les comportements culturels, formuler des hypothèses sur les raisons qui conditionnent les habitudes et les comportements différents ; établir des liens, distinguer les principes organisateurs au sein de la culture étrangère. Dans le cadre du cours d'expression théâtrale, l'apprenant-spectateur comme l'apprenant-acteur sont sollicités dans ce travail d'analyse et d'observation du comportement culturel du personnage à jouer : quels sont ses comportements ? Sont-ils étranges, anormaux, différents, suspects ? S'ensuit la formulation d'hypothèses sur les raisons qui conditionnent ces comportements et la capacité de raisonnement de l'interlocuteur par rapport à ces raisons. Ces différentes étapes d'appréciation du comportement culturel prennent place avant le jeu (préparation du contexte culturel), pendant le jeu (coaction sociale des acteurs avec, comme le suggère Goffman, règles, contraintes et normes de la rencontre) et aboutissent à une réflexion partagée après le jeu. Cette phase permet d'analyser non seulement son propre comportement culturel mais aussi celui de l'autre car, dans son jeu, l'individu n'est pas vierge de tout savoir : il dispose d'outils conceptuels dont il n'a pas lieu de remettre en cause l'efficacité puisque ceux-ci ont fonctionné dans son système culturel d'origine. Ainsi, l'apprenant est-il amené à utiliser ses propres réflexes culturels (par exemple : un étudiant américain a facilement tendance à faire coucou de la main lorsqu'il dit «bonjour » en français) ou une sorte de mimétisme culturel (imitation de sa culture ou de la culture étrangère dans laquelle il vit) ou bien fait-il appel à l'impensable culturel ? Cet échange permet à l'apprenant de se décentrer du «Moi», d'entrevoir d'autres classifications de la réalité et de prendre conscience de la nonuniversalité de sa culture.

\section{Conclusion}

L'apprenant est-il capable de porter un regard extérieur sur sa manière de s'exprimer oralement en langue étrangère? A-t-il conscience de pouvoir orchestrer diverses compétences ? Peut-il se détacher des structures et notions apprises hors-contexte ou des fonctions isolées du langage pour s'engager dans des coactions spontanées ? Faudrait-il pour cela lui en proposer. Certes, le CECR invite à la «tâche» mais il est loin de stimuler des pratiques langagières qui impliquent la prise en compte de la multicanalité communicative ; des pratiques qui se détacheraient du linguistique pour faire place au non verbal; des exercices qui prendraient en compte l'engagement de l'apprenant, sa créativité et sa relation à l'autre. 
Certes, le cours d'expression théâtrale propose des activités fictives et peu authentiques - qu'est-ce qui, de toute façon, est réellement authentique dans l'espace pédagogique ? - mais il donne à l'apprenant une chance de découvrir la langue à travers le langage en prenant conscience de sa voix, de son corps et aussi de la multiplicité des ressources qui existent au cours d'un acte communicatif: la présence, l'engagement, l'attitude, la demande de précisions, le geste et enfin... l'autre. L'activité théâtrale ne saurait se construire seule : elle a besoin du jeu de l'autre pour avancer. L'apprenant-acteur a besoin $\mathrm{du}$ regard de l'apprenant-spectateur pour se regarder lui-même, observer ses propres habitudes culturelles, les analyser, s'étonner de ne pas les avoir perçues ainsi auparavant, les frotter aux autres et ainsi, redécouvrir l'autre. La réflexion autour de la langue et du langage, donc de la culture, ne fait pas seulement suite à l'acte communicatif : elle en est aussi le déclencheur.

\section{Bibliographie}

Busson, V. «Utiliser l'improvisation contextualisée ». Le français dans le monde 256 (1993). 55-59.

Caré, J.-M. «Apprendre les langues étrangères autrement», propos recueillis par Jacques Pêcheur. Le français dans le monde 302 (1999). 48-49.

Conseil de l'Europe/Strasbourg. Un cadre européen commun de référence pour l'apprentissage et l'enseignement des langues. Paris : Didier, 2001.

Lubart, T. Psychologie de la créativité. Paris : Colin, 2003.

Payet, A. Activités théâtrales en classe de langue. Paris : Clé international, 2010.

Perrenoud, $\mathrm{P}$. Texte d'une conférence au Colloque «Le développement des compétences en didactique des langues romanes », Louvain-la-Neuve, 27-23 janvier 2000.

Pierra, G. Le corps, la voix, le texte. Paris : Harmattan, 2006.

Programme Socrates. Le français par les techniques théâtrales. Cahier d'exercices, 2001.

Puren, C. «Perspectives actionnelles et perspectives culturelles en didactiques des languescultures : vers une perspective co-actionnelle, co-culturelle ». Les Langues modernes (juil.-aout-sept 2002). 55-71.

Tabensky, A. Spontanéité et interaction : le jeu de rôle dans l'enseignement des langues étrangères. Paris : Harmattan, 1997.

Tournier, C. Manuel d'improvisation théâtrale. Np : L'eau Vive, 2003.

Vielmas, M. À haute voix. Paris : Clé international, 1991. 
Winkin, Y. La nouvelle communication. Paris : Seuil, 1981.

NOTES

${ }^{1}$ Cf. un des résultats de ces réflexions sous la forme d'un manuel publié en 2011, Clé International.

${ }^{2}$ Dorénavant CECR.

${ }^{3}$ Par «correction langagière », nous entendons une correction qui dépasse les éléments linguistiques et s'intéresse aux éléments paralinguistiques.

${ }^{4}$ Cf. 3.3.

${ }^{5}$ Gisèle Pierra envisage la langue comme lieu de subjectivité et entend l'esthétisme comme l'art de produire des émotions par le rapport actif aux paroles d'une œuvre mise en situation.

${ }^{6}$ L'improvisation contextualisée en classe de FLE : le cas d'apprenants japonais. Thèse soutenue par l'auteure, U de Nantes, 2007. 